\title{
Über die Giftigkeit einiger Harmanderivate für Regenwürmer.
}

\author{
Von \\ Chikasato Kadoyama. \\ (門 山 周 琴) \\ (Aus dem Pharmakologischen Institut der Tohoku Reichsuniversität \\ zu Sendai. Divektor: Prof. Dr. S. Tagi.)
}

Dass die anthelmintische Wirkung der Früchte der Steppenraute, welche von gewissen orientalischen Völkern von alters her als Wurmmittel verwendet werden, auf ihrem Gehalt an Harmalin beruht, wurde von Flu ry ${ }^{1 /}$ wahrscheinlich gemacht, indem er fand, dass das Alkaloid in einer verdünnten Lösung auf die Askariden des Schweines eine schüdliche Wirkung zeigte. Dies wurde von Seto ${ }^{2}$ bestätigt, welcher nachwies, dass das Alkaloid eine santoninartige Wirkung auf abgeschnittene Regenwurmsegmente ausübte. Es scheint nicht nur in theoretischer, sondern auch in praktischer Hinsicht von Interesse zu sein, festzustellen, ob dem Harman und seinen Derivaten auch dieselbe Wirkung zukommt, und wenn dies der Fall ist, ihre Wirksamkeit zu bestimmen. Zu diesem Zwecke ist es geeignet, Regenwürmer als Versuchstiere zu gebrauchen, da sie, wie oben gesagt, eine deutliche Reaktion auf die Harmalindarreichung zeigen und von $\mathrm{Yagi}^{3}$ als für Wertbestimmung von Wurmmitteln verwendbar bewiesen wurden, ferner da sie uns sehr leicht und sogar in Unzahl zur Verfügung stehen können.

Meine Versuche wurden im August an Allolobophora foetida, einer Art von Regenwürmern, mit Harman, Harmalan, Harmin und Harmalin angestellt. Diese wurden in Leitungswasser, in welchem das Tier mehrere Tage, ohne bemerkbare Schüden zu erfahren, am Leben bleiben kann, in verschiedenen Konzentrationen gelöst, und darein das Tier eingetaucht, um die Vergiftungserscheinungen zu beobachten. Dabei wurde es ge-
1) Flury, Arch. f. exp. Path. und Pharm, 1911, 64, 105.
2) Seto, Fol. Pharm., Jap., 1929, 9, 150.
3) Yagi, Zeitschr. f. ges. exp. Med., 1914, 3, 64. 
funden, dass diese bei allen genannten Substanzen anscheinend ganz gleich waren.

Kurz nach dem Hineinlegen in die Giftlösung wird das Tier selır unruhig, macht lebhafte Bewegungen, die denen der Moskitolarven analog sind, indem es aus den Rückenporen gelbliches Sekret ausspritzt. Die Bewegungen, sowohl die spontanen, als auch die reflektorischen, fangen aber an, nach einiger Zeit immer schwächer zu werden, bis das Tier sie nicht mehr ausführt. Dabei sieht es ganz schlaff aus und ist auch auf mechanische Reize nicht imstande, selbst lokale Muskelkontraktion herbeizuführen. So ist es klar, dass die betreffenden Substanzen in einer genügender Konzentration die Muskulatur des Regenwurms lähmen.

Die Wirksamkeitsbestimmung unsrer Substanzen wurde nach dem Verfahren von $Y$ a gi ausgeführt, welches darin besteht, dass man eine Serie von 5 Stück Würmern in jedes Gefüss, welches $10 \mathrm{cem}$ der Giftlösungen von verschiedenen Konzentration enthält, einbringt, um dadurch diejenige schwächste Konzentration, die alle 5 Würmer innerhalb 5 Stunden vollständig zu lähmen vermag, aufzusuchen. Und die Versuche ergaben folgendes.

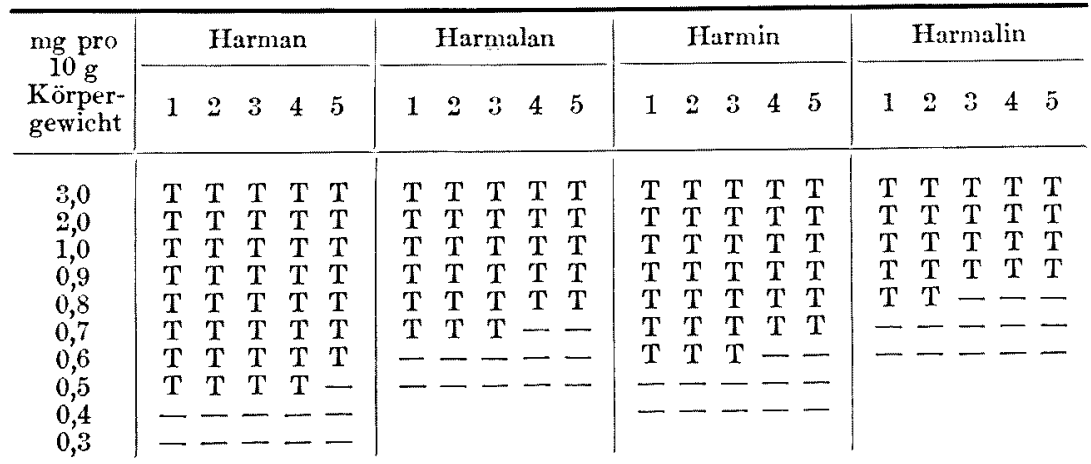

$\mathrm{T}$ bedeutet Tod innerhalb 5 Stunden.

- bedeutet Amlebenbleiben am Ende der 5. Stunde.

Aus dieser Tabelle ist ersichtlich, dass die verschiedenen untersuchten Substanzen, wenn auch nicht sehr grosse, doch deutliche Unterschiede in der Giftigkeit für Regenwürmer aufweisen. Von ihnen wirkt das Harman am stärksten und das Harmalin am schwächsten, und von den zwei übrigen ist das Harmin etwas stärker wirksam als das Harmalan.

Es scheint bemerkenswert zu sein, dass das Harman, welches bei Mäusen die schwächste krampferregende Wirkung aufweist, für Regenwürmer 
am giftigsten ist. Dass das Harmin bzw. Harmalin als das Harman bzw. Harmalan und auch das Harmalin bzw. Harmalan als das Harman bzw. Harmin für Regenwürmer weniger giftig ist, beweist, dass die muskellähmende Wirkung des Harmans sowohl durch das Einführen der Methoxylgruppe in die 11-Stellungen der Harmanstruktur als auch durch die Hydrierung die 5- und 6-Stellungen derselben abgesch wächt werden. Vergleicht man diese Beziehungen zwischen der pharmakologischen Wirkung und chemischen Konstitution mit denjenigen, die man bei den Versuchen an Mäusen und Fröschen kennen gelernt hat, so findet man eine interessante Tatsache, dass dieselben Seitenketten im Molekül einer Substanz ihre Wirksamkeit je nach ihren Grundwirkungen verschiedenartig beeinflussen. 